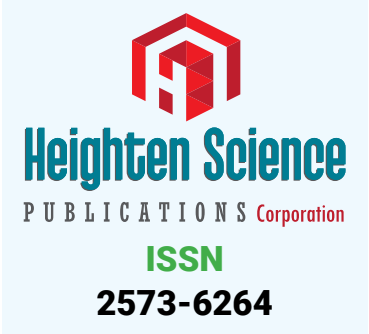

*Address for Correspondence: Andrew Soundy, $\mathrm{PhD}$, University of Birmingham, Birmingham, B15 2TT, UK, Email: A.A.Soundy@bham.ac.uk

Submitted: 16 September 2017

Approved: 02 October 2017

Published: 03 October 2017

Copyright: @ 2017 Pidgeon L, et al. This is an open access article distributed under the Creative Commons Attribution License, which permits unrestricted use, distribution, and reproduction in any medium, provided the original work is properly cited.

Keywords: Telerehabilitation; Tele rehabilitation; Telemedicine; E-health; MS; Multiple sclerosis;

Physiotherapy; Physical therapy; Rehabilitation
Review Article

\section{Determining the use and value of social}

support in Telerehabiliation Interventions
for individuals with Multiple Sclerosis: A narrative synthesis review

\author{
Lucinda Pidgeon ${ }^{1}$, Trudy Pelton ${ }^{2}$ and Andrew Soundy ${ }^{3 *}$ \\ ${ }^{1} \mathrm{BSc}$, University of Birmingham, Birmingham, B15 2TT, UK \\ ${ }^{2} \mathrm{PhD}$, Therapy, University Hospitals Birmingham, B15 2TH, UK \\ ${ }^{3} \mathrm{PhD}$, University of Birmingham, Birmingham, B15 2TT, UK
}

\section{Abstract}

Background: Telerehabilitation has been identified as an effective treatment that promotes exercise rehabilitation in individuals with multiple sclerosis (MS). Social support is recognised as a core element of such interventions. However further research is needed to consider the role and value of different social support domains.

Aim: Review available literature to assess and synthesise the use and value of social support within telerehabilitation interventions for individuals with MS.

Method: A narrative synthesis was conducted. A systematic search of included articles was conducted. Electronic databases were searched from inception to January 2017. Other search methods were undertaken. Evaluation and synthesis of included articles utilised risk of bias assessment and a 4-stage synthesis process.

Main Results: A total of fourteen studies, involving 718 participants (505 female, 213 male; aggregated mean age 47.6 years) with MS, were included. Esteem support was the most frequently reported method of social support, followed by informational, emotional and tangible. It would appear social support can be beneficial in assisting participants to adhere to treatment interventions. Visual feedback may be directly beneficial to improve impaired balance in individuals with MS. A model for future interventions is provided.

Conclusions: Social support appears to increase the adherence of participants with MS to telerehabilitation interventions. Unique findings provide an indication for the direction and content of future interventions. Further research is necessary to ascertain the optimal types and frequencies of social support delivery and its effect on health outcomes for participants with MS.

\section{Introduction}

\section{Multiple sclerosis}

Multiple Sclerosis (MS) is a chronic, neurological condition, characterised by demyelination in the central nervous system. Motor weakness, fatigue and impaired mobility are symptoms of the disease [1]. The symptoms typically worsen with disease progression, for example after 15 years of being diagnosed with MS 58\% of patients report noticeably impaired mobility, which increases to $82 \%$ following 30 years of disease duration. This inhibits the function and participation of the diagnosed individual, as they are unable to achieve their normal tasks without assistance, or may be forced to give up some activities entirely. MS populations consistently report low levels of physical activity [2] and poor adherence to treatment interventions [3,4]. These two factors are closely associated to increased morbidity and mortality [5]. It is therefore crucial that an effective treatment intervention is established, to assist the patient to manage their symptoms effectively and to remain as functional as possible. 


\section{Telerehabilitation}

Physiotherapy and exercise has long been acknowledged as beneficial for MS symptoms. It facilitates an increase in physical function and independence by improving physical parameters such as muscular power, endurance and reducing fatigue [6-8]. Telerehabilitation is defined as "the use of information and communication technologies as a medium for the provision of rehabilitation services to sites or patients that are at a distance from the provider" [9].It is considered a form of rehabilitation. Telerehabilitation offers an excellent platform in which to provide necessary physiotherapy interventions to patients who have limited access to transport or inhabit very remote areas [10]. Much of the current literature explores its use in stroke patients (e.g.,[11]), however the rehabilitation method is now being implemented across a broader range of patient groups, including MS $[9,12]$. To the best of the author's knowledge, there is no available literature denoting the value of social support in telerehabilitation interventions.

\section{Social support}

Social support is the provision of resources intended to enhance an individuals' well-being. Past research has defined social support domains as functional social support (emotional, tangible, information, or esteem) and structural social support (group processes) $[13,14]$. Social support has traditionally been found to be essential in rehabilitation interventions. For instance, it can provide motivation of individuals with MS to adhere to an intervention [15]. Emotional and esteem support can give patients a sense of empowerment and increased confidence [16-18]. Particular techniques like health coaching and motivational interviewing can enhance adherence to rehabilitation interventions. The delivery of timely and accurate informational support can improve emotional well-being and increase physical activity [19-21]. Literature has highlighted the indirect, positive effect that social support has on physical activity in MS participants, through increasing levels of self-efficacy [22,23]. MS patients typically rely heavily upon health professionals to provide this support [21]. However, review evidence $[24,25]$ has identified poor interactions between patients with MS and health care professionals.

As telerehabilitation is a form of rehabilitation that utilises similar social support techniques used in mainstream rehabilitation such as health coaching, providing personalised information, telephone counselling and motivational interviewing, research is needed to consider whether telerehabilitation interventions provide similar benefits to patients as identified in mainstream rehabilitation [26]. One way of establishing the impact of different domains of social support on health-related outcomes from telerehabilitation could be achieved, is through the use of a specialist review. For instance, an approach such as a narrative synthesis that is able to identify elements of the intervention that are attributed directly or indirectly to the results obtained. For instance, our group recently developed such a review that could be used for such a purpose [27]. Such an approach would be a unique and valuable first step in providing a new solution to inactivity and lack of adherence to interventions in people with MS. Thus the purpose of this review was to examine the role and value of social support in telerehabilitation interventions for individuals with MS. To the best of the author's knowledge there is no past review which has investigated this.

\section{Methodology}

A subtle realist paradigm was adopted for this review [28]. This paradigm enables research to consider central and key features from investigation. The approach does not claim to identify a single truth; rather it considers a reality, which many can appreciate with likely pragmatic implications generated.

A narrative synthesis review [29], was undertaken in 3 distinct stages of the process are as follows: 1) "developing a theory", 2) "developing a preliminary synthesis", 3) "exploring relationships" and 4) "assessing the robustness of the synthesis". 


\section{Developing a preliminary synthesis}

A comprehensive search of the available literature was performed to identify studies examining the use of telerehabilitation in MS populations. Data on the types of social support used, the providers of the support and whether study outcomes are accredited to the social support implemented, was collected and presented in tables in the appendix of the synthesis.

\section{Search strategy}

A systematic search of the available literature was conducted Electronic searches of the following databases from inception to January 2017, were performed; Web of Science, AMED, EMBASE and CINAHL. Each search strategy combined key terms for the type of intervention and the population. The following key terms were used: 'telerehabilitation', 'tele rehabilitation', 'telemedicine', 'e-health', 'MS', 'multiple sclerosis', 'physiotherapy', 'physical therapy' and 'rehabilitation'. Standard Boolean operators were utilised. Citation chasing of review articles [31,32] and included articles was completed in addition to this. Searches were also made on Google scholar and by examining the research profiles of lead authors from the included studies.

\section{Selection criteria for the review}

The SPIDER acronym [30], was used to create the eligibility criteria for this review, which is listed below:

(S) Sample - individuals with a diagnosis of MS. Any studies without participants with MS were excluded. Studies with mixed samples were included if separate analysis on individuals with MS was provided.

(PI) Phenomenon of Interest - studies must use a telerehabilitation intervention to promote physical activity or exercise, either as the sole intervention or in conjunction with other methods. Studies must reference at least 1 of the 4 dimensions of social support [27], in their methods section. This allows for comparisons between studies so the synthesis can be useful. Additionally, studies must assess a change in participants from pre-intervention to post-intervention.

(D) Design - a variety of study design methods were included; randomised controlled trials, pre-experimental designs and randomised cross-over trials. Qualitative methodologies, case proceedings, case reports and fiction-based work were excluded. The reason for this was to ensure the studies assessed the effectiveness of the intervention.

(E) Evaluation - subjective interpretation of the use and value of social support in the articles, in addition to all outcome measures used in the studies, with the aim of establishing associations between the two.

(R) Research types - to be included, articles had to include quantitative results denoting the effects of a telerehabilitation intervention. Studies with qualitative data only were excluded.

Other criteria: all articles had to be accessible in English.

\section{Study selection process}

The primary author screened identified articles, initially by title and abstract. Articles that could not be irrefutably excluded based solely on the title and abstract, as decided by the primary author and corresponding author were retrieved in full-text. The eligibility criteria was then applied to full-text articles. 


\section{Critical appraisal}

The primary author undertook a critical appraisal assessment of the included studies. The Cochrane Risk of Bias tool was used (https://www.cochrane-handbook. org). This tool was selected because it permits subjective judgement of the bias present in the studies. This allows author to decide which domains are most important within the context of the studies, which is helpful to establish a risk of bias criteria. The tool considers 6 domains, which guides and focuses the risk assessment: Selection bias (random sequence generation and allocation concealment), Performance bias, Detection bias, Attrition bias, Reporting bias and other bias. Studies scored either High, Low or Unclear for each domain, from which a summary of risk of bias was determined. A risk of bias criteria was established. Studies indicating high risk of bias in 6 or more domains, and studies with a high risk of bias in 4 or more domains including both performance and detection bias would be excluded. No studies were excluded based on this criterion.

\section{Synthesis}

Three stages were conducted for the synthesis by the primary author. The primary stage consisted of the identification of the demographics in included studies. This encompassed participants' gender, age and a summary of the intervention. This information is presented in a data extraction form (Supplementary Table 1). The second stage involved the identification of any of the four domains of social support utilised in the studies (Supplementary Table 2), and determining who provided this support. Once the provider of the support was ascertained, vote counting was used to determine the frequency of support providers across the included studies, details of which can be seen in Supplementary Table 3. The third stage categorised the identified social support into different functional domains, which is displayed in Supplementary Table 4. The secondary synthesis undertaken attempted to establish whether any of the study findings can be attributed to the use of social support. This was carried out by the primary author, and is summarised in Supplementary Table 5. A further synthesis was undertaken by the corresponding author, this was to aggregate the direct or implied benefits that social support has on intervention outcomes. The results of this final synthesis are presented in Supplementary Table 6. A proposed model for future telerehabilitation interventions was created from this final stage.

\section{Results}

Results are presented by focusing on the following sections: (1) summary of included studies, (2) critical appraisal of studies, (3) results from the primary synthesis, and (4) results from the secondary synthesis.

\section{Considering the summary of included studies}

One hundred and fifty four articles were initially identified, after applying eligibility criteria 14 articles were included in the narrative synthesis. A full description of the search strategy utilised is demonstrated through a PRISMA flow diagram (Figure 1).

Three study designs were included: eleven randomised controlled trials, two preexperimental designs and one cross-over study. Across the included studies a total of 718 participants (505 [69.5\%] female, 213 male; aggregated mean age 47.6 years) with MS were represented. The inclusion criteria varied considerably between the studies. All studies included participants with definitively diagnosed MS, but only 5 studies required the diagnosis to be defined in accordance with the McDonald criteria, [3336,12]. Nine studies included either a partial or complete breakdown of the subtypes of MS, which identified Relapse-Remitting Multiple Sclerosis (RRMS) as the leading sub-type; (RRMS $n=425$ (85.5\% of reported MS sub-type), Primary Progressive MS (PPMS) $n=22$, Secondary Progressive MS (SPMS) n= 50, Unknown $n=221$ ). 


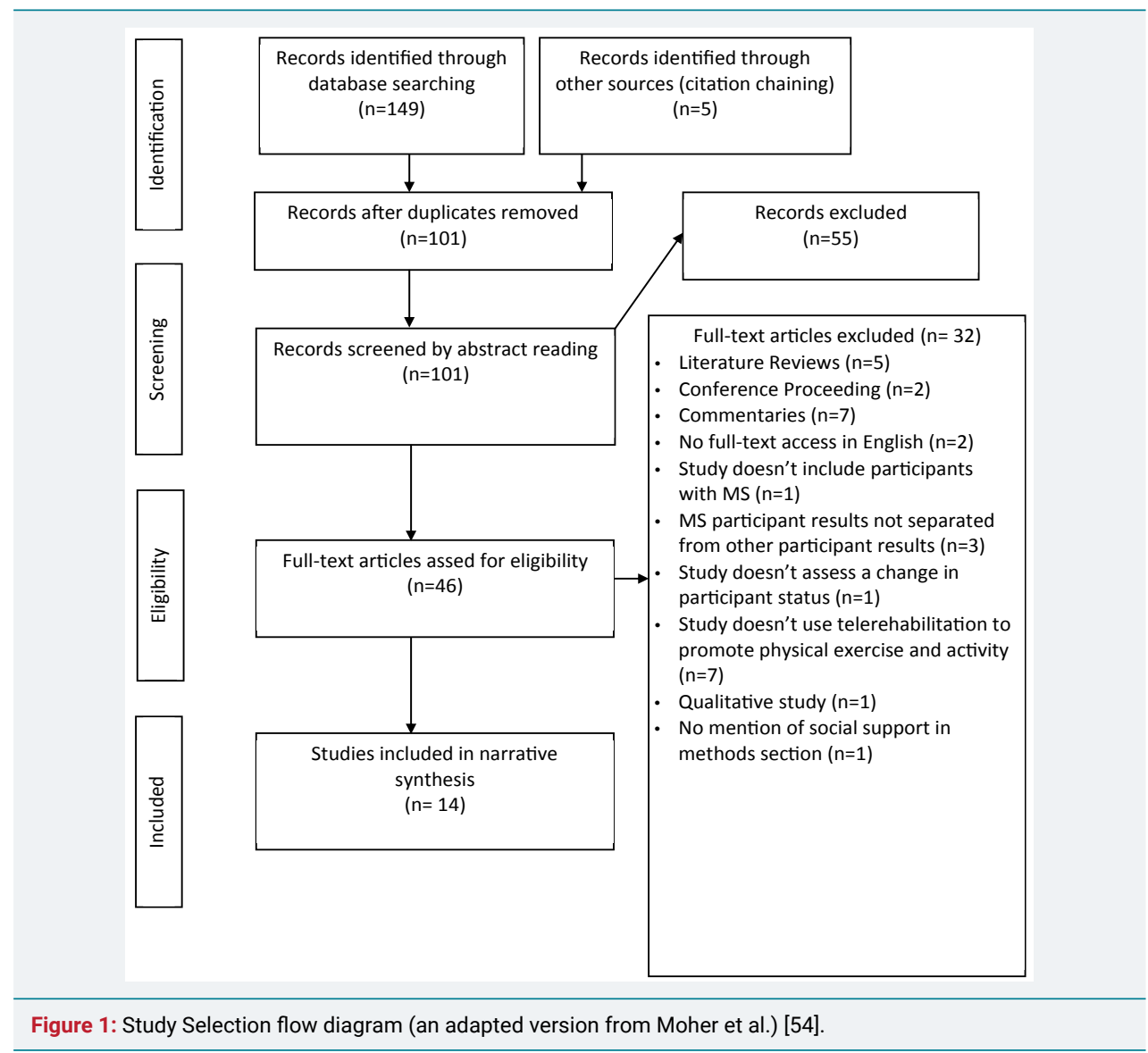

Multiple telerehabilitation interventions were implemented across the studies; Web-based interventions $(n=4)$, Telephone sessions $(n=3)$, Home-based machines $(n=2)$ and Individualised virtual reality telerehabilitation $(n=5), 4$ of which specifically used the Nintendo Wii games console. All telerehabilitation interventions encouraged an increase in physical activity or exercise, either as the main intervention or in conjunction with other methods. Interestingly only one study [37] referenced a peer support component, through an online forum. The predominant intervention setting was at the participant's own homes $(\mathrm{n}=12)$, with only two interventions provided in an outpatient setting. The duration time ranged from 6-24 weeks, with a median and mode of 12 weeks. Many studies lacked an adequately controlled control group; 5 studies used a waiting list control, 2 studies provided participants with information but no program to adhere to and 3 studies lacked a control group entirely. A high completion rate across all the included studies was noted. The greatest participant dropout rate was in Huijgen et al. [38], which lost 11 out of 81 participants (13.58\%), however this was a mixed sample of participants and it was not reported whether the participants who dropped out had a diagnosis of MS, traumatic brain injury or stroke. Detailed information of the included studies' interventions is presented in Supplementary Table 1 in the appendix.

\section{Critical appraisal of included studies}

The criteria for types of bias present ((occurrence within studies / total number of studies) in the studies were; 1 ) Selection bias-random sequence generation $(3 / 14$, 21.4\%), 2) Selection bias-allocation concealment (10/14, 71.4\%), 3) Performance bias $(14 / 14,100 \%), 4)$ Detection bias $(1 / 14,7.1 \%), 5)$ Attrition bias $(2 / 14,14.2 \%)$, 6) Reporting bias $(1 / 14,7.1 \%), 7)$ Other bias $(11 / 14,78.6 \%)$ due to an absence of published protocols. Many studies (5/14, 35.7\%) contained unclear, or inadequately reported blinding of the assessors. The most common type of bias was Performance bias, present in all studies, due to the inability of the studies to blind participants. All the included studies contained at least 1 domain that scored high for risk of bias. 


\begin{tabular}{|c|c|c|c|c|c|c|c|c|c|}
\hline \multirow[t]{2}{*}{ Study } & \multicolumn{7}{|c|}{$\begin{array}{l}\text { Component of risk of } \\
\text { bias }\end{array}$} & \multirow[t]{2}{*}{$\begin{array}{l}\text { Summary } \\
\text { within trial }\end{array}$} & \multirow[t]{2}{*}{ Comments on high risk components } \\
\hline & 1 & 2 & 3 & 4 & 5 & 6 & 7 & & \\
\hline $\begin{array}{l}\text { Bombardier } \\
\text { [51] }\end{array}$ & $\mathrm{L}$ & L & $\mathrm{H}$ & $\mathrm{L}$ & $\mathrm{L}$ & $\mathrm{L}$ & $\mathrm{L}$ & $\begin{array}{l}H=1 \\
L=6 \\
U=0\end{array}$ & Performance bias: participants not blinded \\
\hline Brichetto [33] & L & $\mathrm{H}$ & $\mathrm{H}$ & $\mathrm{L}$ & $\mathrm{u}$ & $\mathrm{L}$ & $\mathrm{H}$ & $\begin{array}{l}H=3 \\
L=3 \\
U=1\end{array}$ & $\begin{array}{l}\text { Selection bias: no allocation concealment } \\
\text { Performance bias: participants not blinded } \\
\text { Other bias: no previously published protocol }\end{array}$ \\
\hline Dlugonski [52] & L & $\mathrm{H}$ & $\mathrm{H}$ & U & $\mathrm{L}$ & $\mathrm{L}$ & $\mathrm{H}$ & $\begin{array}{l}H=3 \\
L=3 \\
U=1\end{array}$ & $\begin{array}{l}\text { Selection bias: no allocation concealment } \\
\text { Performance bias: participants not blinded } \\
\text { Other bias: no previously published protocol. }\end{array}$ \\
\hline $\begin{array}{l}\text { Finkelstein } \\
\text { [34] }\end{array}$ & $\mathrm{H}$ & $\mathrm{H}$ & $\mathrm{H}$ & U & L & $\mathrm{L}$ & $\mathrm{H}$ & $\begin{array}{l}H=4 \\
L=2 \\
U=1\end{array}$ & $\begin{array}{l}\text { Selection bias: no randomisation, no allocation } \\
\text { concealment } \\
\text { Performance bias: participants not blinded } \\
\text { Other bias: no previously published protocol }\end{array}$ \\
\hline Huijgen [38] & $\mathrm{U}$ & $\mathrm{H}$ & $\mathrm{H}$ & U & L & $\mathrm{L}$ & $\mathrm{H}$ & $\begin{array}{l}H=3 \\
L=2 \\
U=2\end{array}$ & $\begin{array}{l}\text { Selection bias: no allocation concealment } \\
\text { Performance bias: participants not blinded } \\
\text { Other bias: no previously published protocol }\end{array}$ \\
\hline Mercier [39] & L & $\mathrm{H}$ & $\mathrm{H}$ & $\mathrm{L}$ & $\mathrm{H}$ & $\mathrm{H}$ & $\mathrm{H}$ & $\begin{array}{l}H=5 \\
L=2 \\
U=0\end{array}$ & $\begin{array}{l}\text { Selection bias: no allocation concealment } \\
\text { Performance bias: participants not blinded } \\
\text { Attrition bias: reasons not documented for participants } \\
\text { lost to follow-up } \\
\text { Reporting bias: multiple missing P-values for } \\
\text { experimental group satisfaction outcomes } \\
\text { Other bias: no previously published protocol }\end{array}$ \\
\hline Motl [37] & $\mathrm{U}$ & L & $\mathrm{H}$ & $\mathrm{H}$ & L & $\mathrm{L}$ & $\mathrm{H}$ & $\begin{array}{l}H=3 \\
L=3 \\
U=1\end{array}$ & $\begin{array}{l}\text { Performance bias: participants not blinded } \\
\text { Detection bias: assessors not blinded } \\
\text { Other bias: no previously published protocol. }\end{array}$ \\
\hline Nilsgård [35] & L & L & $\mathrm{H}$ & $\mathrm{L}$ & L & $\mathrm{L}$ & $\mathrm{L}$ & $\begin{array}{l}H=1 \\
L=6 \\
U=0\end{array}$ & Performance bias: participants not blinded \\
\hline $\begin{array}{c}\text { Ortiz-Gutiérrez } \\
\text { [36] }\end{array}$ & $\mathrm{H}$ & $\mathrm{H}$ & $\mathrm{H}$ & $\mathrm{L}$ & L & $\mathrm{L}$ & $\mathrm{H}$ & $\begin{array}{l}H=4 \\
L=3 \\
U=0\end{array}$ & $\begin{array}{c}\text { Selection bias: not completely randomised, no allocation } \\
\text { concealment } \\
\text { Performance bias: participants not blinded } \\
\text { Other bias: no previously published protocol }\end{array}$ \\
\hline Paul [8] & L & $\mathrm{H}$ & $\mathrm{H}$ & $\mathrm{U}$ & L & $\mathrm{L}$ & $\mathrm{H}$ & $\begin{array}{l}H=3 \\
L=3 \\
U=1\end{array}$ & $\begin{array}{l}\text { Selection bias: no allocation concealment } \\
\text { Performance bias: participants not blinded } \\
\text { Other bias: no previously published protocol }\end{array}$ \\
\hline Pilutti [53] & $\mathrm{L}$ & $\mathrm{H}$ & $\mathrm{H}$ & $\mathrm{U}$ & L & L & $\mathrm{H}$ & $\begin{array}{l}H=3 \\
L=3 \\
U=1\end{array}$ & $\begin{array}{l}\text { Selection bias: no allocation concealment } \\
\text { Performance bias: participants not blinded } \\
\text { Other bias: no previously published protocol }\end{array}$ \\
\hline Plow [40] & $\mathrm{H}$ & $\mathrm{H}$ & $\mathrm{H}$ & U & $\mathrm{H}$ & L & $\mathrm{H}$ & $\begin{array}{l}H=5 \\
L=1 \\
U=1\end{array}$ & $\begin{array}{c}\text { Selection bias: no randomisation, no allocation } \\
\text { concealment } \\
\text { Performance bias: participants not blinded } \\
\text { Attrition bias: no reasons provided for } 5 \text { participants } \\
\text { drop-outs } \\
\text { Other bias: no previously published protocol }\end{array}$ \\
\hline Prosperini [12] & $\mathrm{L}$ & $\mathrm{H}$ & $\mathrm{H}$ & L & L & L & L & $\begin{array}{l}H=2 \\
L=5 \\
U=0\end{array}$ & $\begin{array}{l}\text { Selection bias: no allocation concealment } \\
\text { Performance bias: participants not blinded }\end{array}$ \\
\hline Turner [41] & L & $\mathrm{L}$ & $\mathrm{H}$ & L & L & L & $\mathrm{H}$ & $\begin{array}{l}H=2 \\
L=5 \\
U=0\end{array}$ & $\begin{array}{l}\text { Performance bias: participants not blinded } \\
\text { Other bias: no previously published protocol }\end{array}$ \\
\hline
\end{tabular}

Note: Studies denoted by first author. Risk of bias criteria: 1) Selection bias-random sequence generation, 2) Selection bias-allocation concealment, 3) Performance bias-blinding of participants and personnel, 4) Detection bias-blinding of assessors, 5) Attrition bias-incomplete outcome data, 6) Reporting bias-selective outcome reporting, 7) Other biasthreats to the validity of the results. Level of risk: $\mathrm{H}$ ) high risk of bias, L) low risk of bias, $\mathrm{U}$ ) unclear risk of bias.

Two studies reported high risk of bias in only 1 domain, performance bias, which as previously mentioned is notoriously difficult to avoid in physiotherapy trials. Overall, 4 studies, $[34,36,39,40]$, exhibited at least 4 types of bias out of a potential 7 . One study [35] scored highly in the risk of bias for both performance and detection bias. This lack of blinding is likely to impact the validity and reliability of the results obtained from this study. An in-depth review of the risk of bias in the included studies can be viewed in table 1.

\section{The primary synthesis}

Provider of social support within each study: The main providers of support 
identified throughout the studies were Research Personnel ( $n=11)$, Telerehabilitation machines $(n=11)$ and HCP staff $(n=10) .12$ of the included studies utilised more than one source to provide social support. Prosperini et al. [12] and Ortiz-Gutiérrez et al. [36] had the greatest number of different providers of support $(n=4)$. Supplementary Table 3 contains a full analysis of support providers per included study.

Types of functional social support utilised by each intervention: There was a total of 169 identified uses of social support across the included studies. Esteem support was the most frequently provided type of support (64/169) representing $37.87 \%$, closely followed by Informational support (59/169, 34.91\%). Emotional support represented $15.98 \%$ of social support uses (27/169). Tangible support was the only identified type of support not present in all included studies ( $n=10 / 14)$, with only 19/169 occurrences (11.24\%). Details of this can be seen in Supplementary table 2 .

A full breakdown of the different domains of social support provided is displayed in Supplementary Table 4.

Esteem support was most frequently provided through i) encouragement during the intervention sessions $(n=33)$. This was mainly provided through verbal encouragements by physiotherapists and research personnel. Other methods included, discussing participant progress, self-monitoring undertaken by the participants, verbal and text-based performance feedback, offering participant choice and adapting the intervention program to suit individual participants. A large amount of encouragement was ii) delivered outside the main intervention sessions $(n=26)$, again with a large focus on participant self-monitoring. Lastly, iii) visual feedback $(n=6)$ was provided from digital screens. This included visual displays of interactive exercises, and online graphs to track progress.

Informational support comprised of; i) informative sessions $(n=13)$ delivered through regular, scheduled sessions and accessible web-based information. This included sessions targeting participant's barriers to physical activity. ii) Exercise prescription and instructions on how to correctly perform exercises $(n=12)$, iii) technical assistance and support $(n=9)$ predominantly focussed around equipment use. Information about other available services was also provided. iv) outside the main intervention sessions $(n=9)$. Four studies supplied reminders and alerts to engage and inform participants about new available content and upcoming assessments. v) Information on the study procedure was provided prior to the study commencement $(\mathrm{n}=9)$ and vi) via text-based information $(\mathrm{n}=7)$ in manuals, brochures, resource books and website content.

Emotional support included; i) support provided during sessions ( $\mathrm{n}=11)$ using four methods; personal delivery, telephone delivery, via video conferencing and webbased support. Three additional sub-types of emotional support were, ii) assistance to access others $(n=9)$, iii) supervision of the intervention $(n=4)$ in person and via the internet, and iv) support provided outside the session ( $n=3$ ), which was provided over telephone calls.

Tangible support was predominantly provided through i) financial assistance $(\mathrm{n}=8)$ with free telephone calls, pre-paid envelopes and rewards and prizes available to participants. ii) Provision of study material $(n=6)$ which was indicated in five studies. Four studies iii) installed the equipment into the participant's homes $(n=4)$. One study [35] iv) provided physical assistance ( $\mathrm{n}=1$ ), where the physiotherapist controlled the Wii remote to maximise intervention intensity.

\section{Thes secondary synthesis}

The attributed benefits of social support on telerehabilitation outcomes are documented in Supplementary Tables 5, 6 and 7. It would appear the provision of social 
support can indirectly improve health outcomes of a telerehabilitation intervention through a number of techniques. Esteem support can directly and indirectly aid participants' adherence to treatment interventions, which is supported by the very high completion rates noted in the included studies. This has been indicated through a range of methods, including direct support from staff $(n=8)$, discussions to tackle participant's barriers to treatment $(n=4)$, and goal setting $(n=3)$. Five studies reported significant increases in levels of physical activity post-intervention, which may be attributed to this esteem support participants received. Esteem support provided by visual feedback from digital screens in telerehabilitation appeared to render direct improvements to participant's balance in the post-intervention evaluations. This was accompanied by a moderate risk of summarised bias. Informational support was found to directly and indirectly benefit participants through an increase in adherence to treatment leading to increases in physical activity. This was delivered in a variety of ways; verbal information, informative web-based sessions, technical support sessions and text-based information e.g. resource books. However, a high risk of bias existed in the summary of accumulated evidence for informational support. A summary of these benefits is provided in figure 2 , which represents a model of the most effective social support provision from an accumulation of evidence (see Supplementary file: Table 7).

The model summarises the effective methods of social support used to promote adherence, motivation and outcomes for individuals with MS. Six stages are proposed and are represented as below. Stages 1-3: represent an initial training and support for physical activity or exercise intervention or activity that is going to be undertaken. The aims are to: (a) teach individuals how to use the web-site or app, (b) draw up agreed goals using principles of shared decision making and (c) to provide training related information. Stage 4: This stage is the main support system based on the internet or through an app that provides support. This could take the format of a messaging system, a video contact system. This stage could involve different stakeholders for instance, health care professionals and/or peers. Stage 5-6: These stages include identification of whether or not an individual has met the requirements through documented progress via the Internet or application. Where progress has occurred feedback via the Internet or application is given. If the progress is not sufficient or participant has not engaged with the intervention additional support and support systems can be utilised (e.g., support through motivational interviewing). Support at this stage will be at the level of contact with a health care professional.

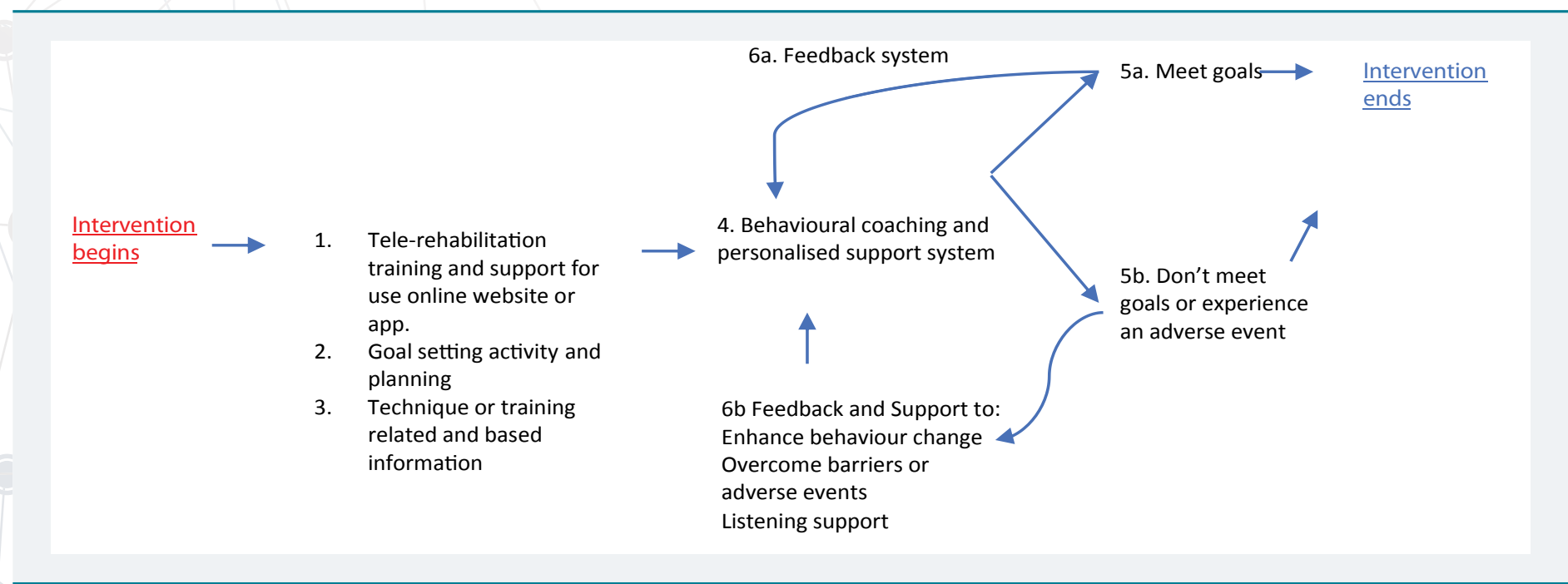

Figure 2: The Proposed Model of Effective Social Support Needed for Telerehabilitation Interventions. 


\section{Discussion}

\section{Main findings}

This is the first study to identify the potential value and benefits of different forms of social support within telerehabilitation for individuals with MS. A total of 14 studies were included in the final synthesis to investigate the significance of social support in conjunction with telerehabilitation interventions to promote or increase levels of physical activity for individuals with multiple sclerosis. There was marked heterogeneity between the included studies with regards to trial characteristics, telerehabilitation interventions and outcome measures used. Additionally, management of the control group greatly differed between studies, from waiting list control groups, typical exercise interventions, to no control group. While a few studies implemented telerehabilitation as an adjunct to other intervention methods, the majority of studies utilised telerehabilitation as the main component. Presently, there is limited evidence of telerehabilitation performed in the multiple sclerosis patient group therefore this review represents a best-evidence synthesis of the available literature. The findings from this narrative synthesis identified the type of social support utilised and its influence on health outcomes in telerehabilitation interventions. It was not able to distinguish optimum types and frequencies of social support delivery.

\section{Providers of social support}

The most frequent providers of support were research personnel and the telerehabilitation technologies, rather than HCPs. This is unsurprising given the reduced patient-professional contact time that typically exists in telerehabilitation interventions. In past literature, patients with chronic diseases, such as MS, have relied on established patient-practitioner relationships as a primary source of social support [21]. Clearly the opportunity to develop meaningful relationships with staff is limited due to the reduced contact time, particularly during home-based interventions. Technological advances have enabled telerehabilitation machines to interact with participants and provide social support [10]. This is demonstrated in the Turner et al. [41], where encouraging statements were imparted to the participants by the telehealth home monitoring system on completion of goals. Continually, the telerehabilitation systems are well equipped to present information on participant's physical performance through graphs and charts. A promising finding from this review is the participant satisfaction with the support they received, despite the reduced patient-practitioner contact. It may be that knowledge that a practitioner is monitoring their progress, and possession of contact details for assistive staff, is sufficient to satisfy the participants support needs. This suggests that telerehabilitation is equipped to provided adequate support for the participants, however further research is required to cement this notion.

\section{Functional types of social support provided}

Esteem support was the most frequently provided support sub-type across all included studies. This was commonly provided through verbal encouragements, discussion of participant progress, personal goals and facilitating participant choice. Through the use of advanced technologies, in conjunction with healthcare professionals and research personnel, telerehabilitation has an extra method that can provide esteem support that is not available in more traditional physiotherapy rehabilitation programs. This offers a possible reason for the large reported use of esteem support. Informational support ensued as the second more frequently identified support sub-type. Informational support, as demonstrated in the results and Supplementary Table 4, can be delivered through a vast variety of techniques. This included verbal information delivered personally, over the internet or telephone, textbased information on the internet and in material resources, and images displaying exercise techniques and performance statistics. Furthermore, this support type is 
easy to provide and to report. These factors could explain the high level of reported informational support. Emotional support was third most commonly provided. It is possible that due to the reduced participant-professional contact reported in the telerehabilitation interventions, that emotional support was less frequently provided and received. Conversely, it may be that emotional support was less sought after, due to the vast amount of esteem and information support provided. As expected, tangible support was the least frequently reported social support sub-type, and absent in four studies. Tangible support is primarily required when an individual's assets are lost, an event which did not occur in any of the studies.

\section{Benefits attributable to social support}

The results from this study found esteem support to be directly and indirectly linked to participant adherence. Goal setting appeared to be a useful technique to motivate participants. This is supported by previous literature [24], which also advocates participant involvement in goal setting to empower the patient to achieve their targets. Although long term goals can have negative consequences if they are too difficult or appear daunting, completion of short term goals can generate feelings of optimism and control of one's own life, which may motivate participants to engage. Provision of esteem support through verbal encouragement and motivation, renders escalated levels of self-efficacy, which is a significant predictor of self-reported health status in people with MS [16]. Raised self-efficacy increases the perceived ability of one to achieve a task or handle a situation. This increases the likelihood of participants to utilise the intervention and complete their program. Furthermore, Motl and Snook [23], found raised self-efficacy is significantly linked to increased levels of physical activity in individuals with MS. This may be a contributing factor to the adherence to interventions involving exercise and physical activity. Increased adherence to interventions should generate greater health improvements in participants, thus esteem support can be indirectly linked to the health improvements attained following a telerehabilitation intervention to promote physical activity and exercise in individuals with MS. In contrast, esteem support can have negative impacts and create participant resentment if delivered in a dominating and intrusive manner. It is therefore important to work with participants on an individual level to ensure the positive benefits of this support are obtained, and the negative consequences are minimised.

This narrative synthesis suggests that visual feedback via digital screens during telerehabilitation can directly improve impaired balance in individuals with MS. The positive findings from the included studies that encompass visual feedback may be attributed to an improvement in the participant's ability to perform appropriate postural reactions in response to the proprioceptive stimuli [12]. Work performed on stroke patients offers an additional explanation. One study suggests that the repetition of exercises and continual visual feedback catalyses the motor learning process, leading to greater motor performances [42]. However, a very small sample of the included studies considered visual feedback, therefore further research is required to evidence how visual feedback can improve balance in participants with MS.

Informational support is an important aspect of healthcare delivery for people with MS. Low levels of perceived provision of information is associated with distress and dissatisfaction with healthcare services [20]. A large qualitative study by Gulick [43], reported low levels of perceived informational support in both male and female participants with MS. As informational support was the $2^{\text {nd }}$ most provided support sub-type across the included studies, it suggests a great improvement in the provision of information for MS participants over the last 20 years. This study proposes informational support can indirectly improve health outcomes in participants through improved adherence to interventions. This is supported by past literature, which found significant associations between informational support and health promotion with consequential increases in physical activity [19] and there are a number of reasons as to 
why this could be. Methley [21], highlighted the benefits of timely information delivery on participants' emotional wellbeing. Half of the included studies provided intervention information prior to study commencement in addition to information throughout the study. This may have proved advantageous by increasing the participants' confidence and reducing feelings of stress as they knew what to expect from the intervention, and could prepare accordingly. Copious technical instruction and directions for exercise execution were provided across all included studies. There is evidence to suggest that the informational support provided was valuable in assisting the participants to utilise the intervention effectively, which may have led to participants experiencing feelings of 'mastery', which motivates them to continue to utilise the intervention. Information addressing personal barriers to activities may provide participants with the tools to overcome difficulties, which reduces the risk of intervention cessation. It is therefore likely that the provision of informational support is useful in increasing physical activity in people with MS, which could lead to functional health improvements as a result.

Despite frequent descriptions of the beneficial aspects of peer support for information exchange and empowerment in participants with MS in the literature $[17,18]$, only one study facilitated peer support through an online forum. There is some research to suggest that emotional support is most effectively provided by similar others [44], as they can connect over shared experiences. An addition of a peer support was suggested in the discussions of three studies, with the potential to increase motivation and provide empathy. It is likely this would be a beneficial adjunct to a telerehabilitation intervention as an additional source of motivation and encouragement, although further research specifically regarding this value of a peer support group is required.

\section{Implications}

The results from this review suggest that patients with MS will benefit from social support provided during telerehabilitation interventions. This is inferred through positive associations between different social support techniques and participant health outcomes, through direct and indirect effects on participant adherence, selfefficacy and motivation. Implications from the current review have been amalgamated to produce clinical recommendations for the provision of social support within telerehabilitation interventions promoting physical activity or exercise for patients with MS. Given the current financial and time pressures facing clinicians, it may initially appear challenging to adequately deliver this support. However, if social support can achieve improvements in patient health outcomes, and consequently alleviate some pressure on NHS resources, then providing the social support is a valuable endeavour. Figure 2 provides a suggestion for the effective design of new telerehabilitation interventions. It should be noted that further research is required to assess and confirm these implications.

\section{Recommendations on the use of esteem support to increase adherence}

Esteem support should be provided for participants engaging in a telerehabilitation intervention. Goal setting, with participant involvement, should be a key feature, a concept which is already used in neurological physiotherapy rehabilitation, albeit inconsistently [24]. Focus should be paid to setting short-term achievable goals to engender feelings of optimism and self-confidence. Patients should have direct involvement to their rehabilitation, and where possible be offered a choice of preferred activity. Frequent use of verbal encouragements is suggested throughout treatment sessions. This can be provided by healthcare professionals, research personal or family and peers. Patients can also be motivated outside of intervention sessions, through the provision of devices, such as logbooks and visual displays of progress e.g. 
pedometers or digital graphs. This enables self-monitoring and assessment of their personal progression through the treatment session, which may be motivational. Where possible, visual feedback via digital screens during individualised virtual reality telerehabilitation is recommended to improve balance in MS participants.

\section{Recommendations on the use of informational support to increase adherence}

A large amount of informational support, in a variety of formats, should be provided. This can help reduce levels of distress amongst participants [20] during intervention sessions. Information, involving training sessions with the telerehabilitation technologies, should be constantly accessible to participants. It may also be beneficial to offer informational support before embarking on independent use of the telerehabilitation method. The opportunity for participants to trial the intervention and work through any difficulties with the technology alongside a trained professional could reduce the likelihood of technical difficulties at a later stage, enhancing participant use. Participants should receive information about optimum exercise execution to improve ease performance. Continually, participants should be informed on the beneficial effects that physical activity can bring them, as this provides reasoning behind why they need to adhere to their treatment and can help participants find motivation. It may be beneficial to offer specific sessions to target overcoming barriers to activities, which would allow them to continue with their rehabilitation. Providing participants with the knowledge and skills to be able to self-monitor their performance and progress is necessary, as this can provide needed motivation and health care professionals are not always available to provide this information.

\section{Limitations}

\section{Methodological limitations}

Numerous methodological limitations of the study were noted. There was significant heterogeneity of the evidence, with regards to a large variety of outcome measures used, use of social support and the diverse telerehabilitation treatment interventions implemented (supervised virtual reality, telephone delivery, web-based delivery). It is challenging to draw conclusions from this vast variety of intervention types and social support delivery, which creates a difficulty when attempting to establish valuable clinical recommendations.

The included studies contained high risks of bias. The majority $(n=10)$ of the included studies contained at least 3 components which ranked high for risk of bias, which included performance bias $(n=14)$ and allocation concealment bias $(n=10)$. Details can be found in table 1. This can have significant consequences to the internal validity of included studies [45]. A lack of double blinding, which was present in all included studies because it was not possible to blind participants to their treatment interventions, can result in significantly exaggerated treatment effects. It should also be noted that 6 of the included studies ranked 'unclear' for detection bias due to inadequate or imprecise reporting of study personnel blinding. While this is a common finding in the literature [46], it can have detrimental implications to the validity of the findings nonetheless. Additionally, treatment effect sizes can be exaggerated where there is an absence of adequate allocation concealment.

The overall sample size of included articles was small $(n=14)$. This can be attributed to the small evidence base existing for telerehabilitation in participants with MS. Additionally, the included articles contained relatively small sample sizes, with an average of 51 participants per study. The implications of this may be imprecise treatment effect sizes, which do not accurately reflect the effect of the intervention [45].

There was a significant lack of long-term follow-up assessments, with only 2 of the studies performing any follow-up assessment at all. This may have disregarded any 
maturation of participants, which if considered, may have affected internal validity [45]. In addition to this, the long-term effects of the treatment interventions remain unknown. For participants with MS, an evolving, progressive disease, it would be valuable to ascertain the participants' capabilities of performing the intervention as their disease progresses.

A large limitation of the included studies is the limited reporting of specific social support techniques used. Five of the studies contain the phrase 'social support', despite the subjectively identified use of social support in all studies (see Supplementary Table 2). Many instances of social support are delivered in a casual, mundane way, which renders them 'invisible' as social support provision, and hence can be over looked. Due to this, not all applications of social support are accurately identified. This may undermine conclusions made.

There may also be a language bias as studies not accessible in English were excluded. Continually, publication bias cannot be ruled out as the search strategy may have missed some potentially valuable results from studies that are yet to be published.

\section{Bias within the data}

There were some threats posed to the internal validity of included studies. Although unavoidable, no participants involved in the studies were blinded, which increases the likelihood of biased assessment outcomes. Poor regulation of the control group activity, as demonstrated in most of the included studies (by a wait-list control group or absence of a control group), poses a significant risk to the treatment fidelity, which again, threatens the internal validity of the study outcomes. Comparison against a waitlist control group, which by nature guarantees that the intervention group receive more rehabilitation, creates a bias in favour of the intervention group. This is because the intervention group are then more likely to achieve improvements as a result of increased intensity and frequency of rehabilitation.

The overall number of women included in the trials greatly exceeded the number of men, with all but two studies comprising of more than $50 \%$ female participants. Although the prevalence of MS is higher in females in the UK according to Mackenzie et al. [47], the exact ratio of females to males is not accurately represented in the studies, and is therefore not a true representation of the MS population. The reporting of participant ethnicity was very poor throughout the studies. The sample populations were predominantly white, non-Hispanic in the studies, which reported data for ethnicity $(n=4)$. The risk factors for MS vary across different ethnicities [48], and an underrepresentation of ethnic minorities in this synthesis would reduce the external validity of results to wider populations than were analysed. Furthermore, all included studies were conducted in the Western hemisphere; therefore this may limit the generalisability of the results to participants from this region.

There was limited information of the participant's sub-type of MS available throughout the included studies. Of the studies, which identified the diagnosed MS sub-type, RRMS was the leading subgroup of MS represented in this synthesis. The categorical differences between subtypes of MS influence the course of the disease, which has implications on an individuals' ability to participate in certain activities. It is therefore not necessarily appropriate to generalise results gained predominantly from RRMS participants across different subgroups of MS, hence the external validity of the results may be compromised.

Many of the included studies had strict inclusion and exclusion criteria, which restricts the external validity of the results. A selection bias may have occurred in 7 studies, which required participants to possess either a coloured television, phone access or Internet access. This may reduce external validity, because participants from lower socioeconomic backgrounds who lack the capacity to finance the equipment may been overlooked. 
Half of the studies excluded potential participants with a mental health condition. Literature demonstrates a high prevalence of psychiatric disorders among the MS population [49], therefore by excluding this group, a large proportion of potential participants would be lost. It also creates a knowledge gap of the treatment effects on this particular group of MS patients. Hence, the studies are not representative of the whole MS population and generalisability of the results is reduced.

\section{Limitations of the synthesis}

To the authors knowledge, this is the first review of its kind. The format of a narrative synthesis enabled the author to collate information from a variety of studies, with diverse treatment interventions, which may not have been possible with another form of review [50]. The primary author, and undergraduate University student, reviewed and synthesised the included studies. The results of the synthesis are limited due to the subjective techniques utilised to identify social support and to analyse the included studies and study outcomes. The overall accuracy of the synthesis may have been improved with contributions from additional authors. Furthermore, researcher bias is likely to have occurred, as all sections on the synthesis were completed by the non-blinded primary author. With respect to this, caution should be taken when acknowledging the results. The synthesis was not able to deduce definite consequences of specific social support techniques, nor were optimum frequencies of social support identified. Further research is need to ascertain this information.

\section{Recommendations for future research}

Additional information about patient preferences of social support is required to establish optimum types and frequencies of social support used within telerehabilitation interventions. Research should be undertaken on whether telerehabilitation technology encompasses adequate sources of social support, with respect to participant satisfaction and how best to implement social support in telerehabilitation in conjunction with current guidelines and policies. Future research should aim to ascertain the direct results of social support on health outcomes following a telerehabilitation intervention to promote physical activity and exercise.

\section{Conclusion}

This review has identified the broad variety of social support used within telerehabilitation interventions for participants with MS. There is some evidence which indicates social support is beneficial to increase levels of adherence to interventions, indirectly resulting in improved health outcomes. Due to the limitations of the included studies, it was difficult to determine the direct impact of social support on the health outcomes of participants. Therefore, more research is required to investigate the value of social support in telerehabilitation interventions on the health outcomes of participants with MS.

(Supplementary Tables 1-7)

\section{References}

1. Kister I, Bacon TE, Chamot E, Salter AR, Cutter GR, et al. Natural History of Multiple Sclerosis Symptoms. Int J MS Care. 2013; 15: 146-158. Ref.: https://goo.gl/ApY1ZC

2. Motl RW, McAuley E, Snook EM. Physical activity and multiple sclerosis: a meta-analysis. Mult Scler. 2005; 11: 459-463. Ref.: https://goo.gl/snMzdV

3. Mostert S, Kesselring J. Effects of a short-term exercise training program on aerobic fitness, fatigue, health perception and activity level of subjects with multiple sclerosis. Mult Scler. 2002; 8: 161-168. Ref.: https://goo.gl/5DLo6K

4. Remington $G$, Rodriguez Y, Logan D, Williamson C, Treadaway K. Facilitating medication adherence in patients with multiple sclerosis. Int J MS Care. 2013; 15: 36-45. Ref.: https://goo.gl/12C1n8 
5. Klauer T, ZettI UK. Compliance, adherence, and the treatment of multiple sclerosis. J Neurol. 2008; 255: 87-92. Ref.: https://goo.gl/AfuP4F

6. Rietberg MB, Brooks D, Uitdehaag BMJ, Kwakkel G. Exercise therapy for multiple sclerosis. Cochrane Database Syst Rev. 2005; Ref.: https://goo.gl/oWZpiR

7. Khan $\mathrm{F}$, Turner-Stokes $\mathrm{L}, \mathrm{Ng} \mathrm{L}$, Kilpatrick $\mathrm{T}$, Amatya B. Multidisciplinary rehabilitation for adults with multiple sclerosis. Cochrane Database Syst Rev. 2007; 2: Ref.: https://goo.gl/728cr7

8. Campbell E, Coulter EH, Mattison PG, Miller L, McFadyen A, et al. Physiotherapy rehabilitation for people with progressive multiple sclerosis: a systematic review. Arch Phys Med Rehabil. 2016; 97 141-151. Ref.: https://goo.gl/UgM5vs

9. Khan F, Amatya B, Kesselring J, Galea M. Telerehabilitation for persons with multiple sclerosis. Cochrane Database Syst Rev. 2015; 4: Ref.: https://goo.gl/Ua7suS

10. Brennan DM, Mawson S, Brownsell S. Telerehabilitation: enabling the remote delivery of healthcare, rehabilitation, and selfmanagement. Stud Health Technol Inform. 2009; 145: 231-248. Ref.: https://goo.gl/G3iQcA

11. Laver KE, Schoene D, Crotty M, George S, Lannin NA, et al. Telerehabilitation services for stroke. Cochrane Database Syst Rev. 2014; 12: Ref.: https://goo.gl/beYiHf

12. Prosperini L, Leonardi L, De Carli P, Mannocchi ML, Pozzilli C. Visuo-proprioceptive training reduces risk of falls in patients with multiple sclerosis. Mult Scler. 2010; 16: 491-499. Ref.: https://goo.gl/6acn5D

13. Rees T, Hardy L. An Investigation of the social support experiences of high-level sports performers. The Sport Psychologist. 2000; 14: 327-347. Ref.: https://goo.gl/unFYcc

14. Rees T, Smith B, Sparkes AC. The influence of social support on the lived experiences of spinal cord injured sportsmen. The Sport Psychologist. 2003; 17: 135-156. Ref.: https://goo.gl/uSPoHZ

15. McAuley E, Motl RW, Morris KS, Hu L, Doerksen SE, et al. Enhancing physical activity adherence and well-being in multiple sclerosis: a randomised controlled trial. Mult Scler. 2007; 13: 652-659. Ref.: https://goo.gl/UbrLUT

16. Ashford S, Edmunds J, French DP. What is the best way to change self-efficacy to promote lifestyle and recreational physical activity? A systematic review with meta-analysis. $\mathrm{Br} \mathrm{J}$ Health Psychol. 2010; 15: 265-288. Ref.: https://goo.gl/MpxAMX

17. Skår ABR, Folkestad H, Smedal T, Grytten N. "I refer to them as my colleagues": the experience of mutual recognition of self, identity and empowerment in multiple sclerosis. Disabil Rehabil. 2014; 36 672-677. Ref.: https://goo.gl/xLu5Zu

18. Fakolade A, Finlayson M, Plow M. Using telerehabilitation to support people with multiple sclerosis: A qualitative analysis of interactions, processes, and issues across three interventions. British Journal of Occupational Therapy. 2017; 80: 259-268. Ref.: https://goo.gl/FvVALN

19. Ennis M, Thain J, Boggild M, Baker GA, Young CA. A randomized controlled trial of a health promotion education programme for people with multiple sclerosis. Clin Rehabil. 2006; 20: 783-793. Ref.: https://goo.gl/3ZxfKU

20. Malcomson KS, Lowe-Strong AS, Dunwoody L. What can we learn from the personal insights of individuals living and coping with multiple sclerosis? Disabil Rehabil. 2008; 30: 662-674. Ref.: https://goo.gl/Rt2VTN

21. Methley AM, Chew-Graham C, Campbell S, Cheraghi-Sohi S. Experiences of UK health-care services for people with Multiple Sclerosis: a systematic narrative review. Health Expect. 2015; 18: 18441855. Ref.: https://goo.gl/PBHKXx

22. Motl RW, Snook EM, McAuley E, Scott JA, Douglass ML. Correlates of Physical Activity Among Individuals with Multiple Sclerosis. Ann Behav Med. 2006; 32: 154-161. Ref.: https://goo.gl/FCreEs

23. Motl RW, Snook EM. Physical activity, self-efficacy, and quality of life in multiple sclerosis. Ann Behav Med. 2008; 35: 111. Ref.: https://goo.gl/qZjFVs

24. Rose A, Rosewilliam S, Soundy A. Shared decision making within goal setting in rehabilitation settings: A systematic review. Patient Educ Couns. 2017; 100: 65-75. Ref.: https://goo.gl/QYoJmn

25. Soundy A, Roskell C, Adams R, Elder T, Dawes H. Understanding health care professional interactions in multiple sclerosis: A systematic review and thematic synthesis, OJTR. 2016; 4: 187-217. Ref.: https://goo.gl/PXY5tK 
26. Rieckmann P, Boyko A, Centonze D, Elovaara I, Giovannoni G, et al. Achieving patient engagement in multiple sclerosis: A perspective from multiple sclerosis in the $21^{\text {st }}$ century steering group. Multiple Sclerosis and Related Disorders, 2015; 4: 202-218. Ref.: https://goo.gl/DFPbD7

27. Gross J, Vancampfort D, Stubbs B, Gorczynski P, Soundy A. A narrative synthesis investigating the use and value of social support to promote physical activity among individuals with schizophrenia. Disabil Rehabil. 2016; 38: 123-150. Ref.: https://goo.gl/qKWsny

28. Soundy A, Roskell C, Elder T, Collette J, Dawes H. The psychological processes of adaptation and hope in patients with multiple sclerosis: A thematic synthesis. OJTR. 2016; 4: 22-47. Ref.: https://goo.gl/cWZWUd

29. Rodgers M, Sowden A, Petticrew M, Arai L, Roberts H, Britten N, al e. Testing methodological guidance on the conduct of narrative synthesis in systematic reviews. Evaluation 2009;15:49-74.

30. Cooke, A., Smith, D., Booth, A. (2012). Beyond PICO: the SPIDER tool for qualitative evidence synthesis. Qualitative Health Research, 22: 1435-1443.

31. $\mathrm{Yu} \mathrm{CH}$, Mathiowetz V. Systematic review of occupational therapy-related interventions for people with multiple sclerosis: Part 1. Activity and participation. Am J Occup Ther. 2014; 68: 27-32. Ref.: https://goo.gl/Wv8gDc

32. Donzé C. Update on rehabilitation in multiple sclerosis. Presse Médicale, 2015; 44: 169-176. Ref.: https://goo.gl/Qobwys

33. Brichetto G, Spallarossa P, de Carvalho MLL, Battaglia MA. The effect of Nintendo ${ }^{\circledR}$ Wiiß on balance in people with multiple sclerosis: a pilot randomized control study. Mult Scler. 2013; 19: 1219-1221. Ref.: https://goo.gl/oDYVW8

34. Finkelstein J, Lapshin O, Castro H, Cha E, Provance PG. Home-based physical telerehabilitation in patients with multiple sclerosis: a pilot study. J Rehabil Res Dev. 2008; 45: 1361-1374. Ref.: https://goo.gl/Gh2aZg

35. Nilsagård $Y E$, Forsberg AS, von Koch $L$. Balance exercise for persons with multiple sclerosis using Wii games: a randomised, controlled multi-centre study. Mult Scler. 2013; 19: 209-216. Ref.: https://goo.gl/yjtpY4

36. Ortiz-Gutiérrez R, Galán del Río F, Cano de la Cuerda R, Diego A, Isabel M, et al. A telerehabilitation program by virtual reality-video games improved balance and postural control in multiple sclerosis patients. NeuroRehabilitation. 2013; 33: 545-554. Ref.: https://goo.gl/vHSbME

37. Motl RW, Dlugonski D, Wójcicki TR, McAuley E, Mohr DC. Internet intervention for increasing physical activity in persons with multiple sclerosis. Mult Scler. 2011;17: 116-128. Ref.: https://goo.gl/ZfEA5P

38. Huijgen BC, Vollenbroek-Hutten MM, Zampolini M, Opisso E, Bernabeu M, et al. Feasibility of a home-based telerehabilitation system compared to usual care: arm/hand function in patients with stroke, traumatic brain injury and multiple sclerosis. J Telemed Telecare. 2008; 14: 249-256. Ref.: https://goo.gl/4tjF68

39. Mercier HW, Ni P, Houlihan BV, Jette AM. Differential Impact and Use of a Telehealth Intervention by Persons with MS or SCl. Am J Phys Med Rehabil. 2015; 94: 987-999. Ref.: https://goo.gl/vk9aVL

40. Plow M, Finlayson M. Potential benefits of Nintendo Wii Fit among people with multiple sclerosis: a longitudinal pilot study. Int J MS Care. 2011; 13: 21-30. Ref.: https://goo.gl/jxfrmw

41. Turner AP, Hartoonian N, Sloan AP, Benich M, Kivlahan DR, et al. Improving fatigue and depression in individuals with multiple sclerosis using telephone-administered physical activity counseling. $J$ Consult Clin Psychol. 2016; 84: 297-309. Ref.: https://goo.gl/pL5b22

42. Saposnik G, Mamdani M, Bayley M, Thorpe KE, Hall J, et al. Effectiveness of Virtual Reality Exercises in STroke Rehabilitation (EVREST): rationale, design, and protocol of a pilot randomized clinical trial assessing the Wii gaming system. Int J Stroke. 2010; 5: 47-51. Ref.: https://goo.gl/23efWm

43. Gulick EE. Social support among persons with multiple sclerosis. Research in Nursing \& Health. 1994; 17: 195-206. Ref.: https://goo.gl/DdmuiB

44. Hwang JE, Cvitanovich DC, Doroski EK, Vajarakitipongse JG. Correlations between quality of life and adaptation factors among people with multiple sclerosis. Am J Occup Ther. 2011; 65: 661-669. Ref.: https://goo.gl/rdNfiw

45. Campbell D, Stanley JC. Experimental and quasi-experimental designs for research, Chicago: Rand McNally College Publishing. 1963. 
46. Rodgers M, Sowden A, Petticrew M, Arai L, Roberts $\mathrm{H}$, et al. Testing methodological guidance on the conduct of narrative synthesis in systematic reviews: effectiveness of interventions to promote smoke alarm ownership and function. Evaluation. 2009; 15: 49-73. Ref.: https://goo.gl/DJdZ8b

47. Mackenzie IS, Morant SV, Bloomfield GA, MacDonald TM, O'riordan J. Incidence and prevalence of multiple sclerosis in the UK 1990-2010: a descriptive study in the General Practice Research Database. J Neurol Neurosurg Psychiatry. 2014; 85: 76-84. Ref.: https://goo.gl/JA4FB9

48. Langer-Gould A, Brara SM, Beaber BE, Zhang JL. Incidence of multiple sclerosis in multiple racial and ethnic groups. Neurology. 2013; 80: 1734-1739. Ref.: https://goo.gl/Jh3pCk

49. Marrie RA, Cohen J, Stuve O, Trojano M, Sørensen PS, et al. A Systematic review of the incidence and prevalence of comorbidity in multiple sclerosis: Overview. Mult Scler. 2015; 21: 263-281. Ref.: https://goo.gl/yh8y8E

50. Edwards J, Kaimal G. Using meta-synthesis to support application of qualitative methods findings in practice: A discussion of meta-ethnography, narrative synthesis, and critical interpretive synthesis. The Arts in Psychotherapy, 2016; 51: 30-35. Ref.: https://goo.gl/AEwXDM

51. Bombardier $\mathrm{CH}$, Cunniffe M, Wadhwani R, Gibbons LE, Blake KD, et al. The efficacy of telephone counseling for health promotion in people with multiple sclerosis: a randomized controlled trial. Arch Phys Med Rehabil. 2008; 89: 1849-1856. Ref.: https://goo.gl/1jx63V

52. Dlugonski D, Motl RW, Mohr DC, Sandroff BM. Internet-delivered behavioral intervention to increase physical activity in persons with multiple sclerosis: sustainability and secondary outcomes. Psychol Health Med. 2012; 17: 636-651. Ref.: https://goo.gl/nh7X99

53. Pilutti LA, Dlugonski D, Sandroff BM, Klaren R, Motl RW. Randomized controlled trial of a behavioral intervention targeting symptoms and physical activity in multiple sclerosis. Mult Scler. 2014; 20 : 594-601. Ref.: https://goo.gl/jZX5Yd

54. Moher D, Liberati A, Tetzlaff J, Altman DG. Preferred Reporting Items for Systematic Reviews and Meta- Analyses: The PRISMA Statement. PLoS Med. 2009; 6: e1000097. Ref.: https://goo.gl/b8q97y

55. Coulter A. Patient engagement--What works? J Ambul Care Manage. 2012; 35: 80-89. Ref.: https://goo.gl/KirR6X

56. Levack WMM, Weatherall M, Hay-Smith EJC, Dean SG, McPherson K, et al. Goal setting strategies to enhance goal pursuit in adult rehabilitation: summary of a cochrane systematic review and metaanlaysis. Eur J Phys Rehabil Med. 2016; 52: 400-416. Ref.: https://goo.gl/yA4TJx

57. Multiple Sclerosis Society UK. MS in the UK. Obtained from the World Wide Web 13/06/2017. 2016 1-3. Ref.: https://goo.gl/D2rX97

58. Soundy A, Elder T. Developing and applying the theory of psychological adapation needs in patients with multiple sclerosis. In R. R. Watson \& Killgore, W. D. S. Nutrition and lifestyle in neurological autoimmune diseases: multiple sclerosis. Academic Press. 2017.

59. Stennett A, De Souza L, Norris M. Physical activity and exercise priorities in community dwelling people with multiple sclerosis: A delphi study. Disabil Rehabil. 2017; 1-8. Ref.: https://goo.gl/UpFfeQ

60. Six PA. Mechanisms linking social ties and support to physical and mental health. J Health Soc Behav. 2011; 52: 145-161. Ref.: https://goo.gl/3UBeU9 